\title{
Entrectinib: A Review in NTRK+ Solid Tumours and ROS1+ NSCLC
}

\author{
James E. Frampton ${ }^{1}$ \\ Accepted: 15 March 2021 / Published online: 19 April 2021 \\ (c) Springer Nature 2021, corrected publication 2021
}

\begin{abstract}
Entrectinib (Rozlytrek ${ }^{\circledR}$ ) is an orally active, CNS-penetrant, small-molecule, selective inhibitor of the tropomyosin receptor tyrosine kinases TRKA/B/C [encoded by the neurotrophic tyrosine receptor kinase (NTRK) genes $N T R K 1 / 2 / 3$, respectively], the proto-oncogene tyrosine-protein kinase ROS1 $(R O S 1)$ and the anaplastic lymphoma kinase gene $(A L K)$. It is approved for the treatment of adults and paediatric patients aged $\geq 12$ years with $N T R K$ fusion-positive $(N T R K+)$ solid tumours and adults with ROS1 fusion-positive ( $R O S 1+$ ) non-small-cell lung cancer (NSCLC). In trials in adults, entrectinib induced clinically meaningful and durable systemic responses in tyrosine kinase inhibitor (TKI)-naïve patients with locally-advanced or metastatic NTRK+ solid tumours or ROS1+ NSCLC, irrespective of the presence or absence of CNS metastases at baseline. Moreover, entrectinib demonstrated substantial intracranial efficacy in patients with baseline CNS metastases. Entrectinb efficacy in paediatric patients was established on the basis of extrapolation of clinical trial data from adults with NTRK+ solid tumours and children and adolescents aged $<21$ years with recurrent or refractory $N T R K+\mathrm{CNS} /$ solid tumours. Entrectinib was generally well tolerated, with a manageable safety profile. Thus, entrectinib expands the range of treatment options for advanced NTRK+ solid tumours and ROSI+ NSCLC, and may be of particular value in patients with existing CNS metastases and those who are at risk of developing CNS metastases.
\end{abstract}

\section{Digital Features for this Adis Drug Evaluation can be found at https://doi.org/10.6084/m9.figshare.14150207}

Entrectinib: clinical considerations in NTRK+ solid tumours and ROS1+ NSCLC

Oral inhibitor of TRKA/B/C, ROS1 and ALK tyrosine kinases

Designed to be active systemically and in the CNS

Demonstrated durable systemic and intracranial efficacy

Generally well tolerated with a manageable safety profile

The manuscript was reviewed by: F. Facchinetti, INSERM U981, Gustave Roussy Cancer Campus, Université Paris Saclay, Villejuif, France; D. Liu, Department of Pharmacy, Memorial Sloan Kettering Cancer Center, New York, NY, USA; A. Russo, Medical Oncology Unit, A.O. Papardo \& Department of Human Pathology, University of Messina, Messina, Italy.

James E. Frampton

demail@springer.com

1 Springer Nature, Private Bag 65901, Mairangi Bay, Auckland 0754, New Zealand

\section{Introduction}

The diagnosis and treatment of solid tumours has firmly entered the era of personalized (or precision) medicine, with optimal patient management requiring molecular profiling aimed at identifying oncogenic driver mutations that are actionable or potential therapeutic targets [1-4]. In this regard, gene rearrangements (fusions) involving the neurotrophic tyrosine receptor kinase (NTRK) genes NTRK1, NTRK2 and NTRK3 [encoding the tropomyosin receptor kinase (TRK) receptors TRKA, TRKB and TRKC, respectively], the c-ros oncogene 1 receptor tyrosine kinase ( $R O S 1$ ) gene [encoding proto-oncogene tyrosine-protein kinase ROS (ROS1)] and the anaplastic lymphoma kinase gene ( $A L K$; encoding the ALK receptor tyrosine kinase) are variously implicated, albeit often infrequently, in a broad range of ( $>40)$ solid tumour types [5]. NTRK fusions, which are thought to be present in up to $1 \%$ of all solid tumours, are found in a wide variety of adult and paediatric tumour types; they are extremely common (incidence $>90 \%$ ) in rare cancer types, such as infantile fibrosarcoma and mammary analogue secretory carcinoma (MASC), but rare (incidence predominantly $<1 \%$ ) in more common cancer types, such as lung, breast and colorectal cancers, and melanomas $[2,6,7]$. NTRK, ROS1 and $A L K$ fusions are present in $0.1-1 \%, 1-2 \%$ and $4-6 \%$ of cases of non-small-cell lung cancer (NSCLC), respectively [8]. 
Among the commercially available, orally active, smallmolecule tyrosine kinase inhibitors (TKIs), larotrectinib (a selective inhibitor of TRKA/B/C [9]) has been approved for the treatment of NTRK fusion-positive (NTRK+) solid tumours and crizotinib (an inhibitor of multiple tyrosine kinases, including ROS1 and ALK [10]) has been approved for the treatment of ROS1 fusion-positive (ROS1+) NSCLC. However, since larotrectinib has been intentionally designed to have limited CNS penetration [9] and crizotinib also has limited brain penetration [10], there is a need for additional treatments with effective intracranial (IC) activity against primary or secondary brain tumours harbouring NTRK or ROSI fusions [11]. Regarding the latter, NSCLC is the most common cause of brain metastases [12]; at the time of diagnosis, brain metastases were present in $50 \%$ of patients with metastatic NTRK+ NSCLC [13] and 20-40\% of patients with metastatic ROS1+ NSCLC [14, 15], according to small retrospective studies. Patients with CNS involvement have a poorer prognosis than those without IC disease [16, 17].

Against this background, the orally active, small-molecule TKI entrectinib (Rozlytrek ${ }^{\circledR}$; a selective inhibitor of TRKA/ $\mathrm{B} / \mathrm{C}$, ROS1 and ALK) has been designed to have systemic activity and also to cross the blood-brain barrier (BBB) and be retained within the CNS $[18,19]$. Entrectinib has been approved for the treatment of adults and paediatric patients aged $\geq 12$ years with $N T R K+$ solid tumours, as well as adults with ROS1+ NSCLC in several countries, including those of the EU (conditionally) [20], the USA (conditionally) [21], and Japan [22]. This article reviews pharmacological, therapeutic efficacy and tolerability data relevant to the use of entrectinib in these patient populations.

\section{Pharmacodynamic Properties of Entrectinib}

Entrectinib is a potent and selective adenosine triphosphate-competitive inhibitor of TRKA ( IC $_{50}$ value of 1.7 $\mathrm{nmol} / \mathrm{L}), \mathrm{TRKB}(0.1 \mathrm{nmol} / \mathrm{L})$, TRKC $(0.1 \mathrm{nmol} / \mathrm{L}), \mathrm{ROS} 1$ $(0.2 \mathrm{nmol} / \mathrm{L})$ and $\operatorname{ALK}(1.6 \mathrm{nmol} / \mathrm{L})$ in vitro [20, 23]. M5, the major and only active circulating metabolite of entrectinib (Sect. 3), showed similar in vitro potency and activity to the parent drug against TRK, ROS1 and ALK [20,21].

In preclinical studies, entrectinib inhibited the growth of human tumour cell lines driven by NTRK1 or $A L K$ fusions $[18,24]$ and that of engineered murine $(\mathrm{Ba} / \mathrm{f} 3)$ cell lines reliant on NTRK1/2/3, ROS1 or ALK fusions for survival $[18,24]$; it was 40 -times more potent at inhibiting the growth of ROS1-dependent $\mathrm{Ba} / \mathrm{f} 3$ cells than crizotinib [24]. Entrectinib also displayed antitumour activity and induced tumour regression in patient-derived xenograft models of multiple tumour types harbouring NTRK, ROS1 or $A L K$ fusions [18, 19, 24, 25]. Notably, entrectinib inhibited the proliferation of cancer cell lines and $\mathrm{Ba} / \mathrm{f} 3$ cells harbouring NTRK1 kinase domain mutations that confer resistance to another TRK inhibitor, including the 'gatekeeper' residue substitution F589L and the xDFG motif substitution G667C [26].

Importantly, entrectinib is a weak P-glycoprotein (P-gp) substrate that can sustain CNS exposure, unlike crizotinib and larotrectinib, which are strong P-gp substrates with poor brain distribution [11]. In particular, entrectinib showed a weaker interaction than crizotinib and larotrectinib with P-gp in an in vitro assay using transfected porcine kidney epithelial cells overexpressing human P-gp, based on noticeably lower 'apical efflux ratio' (AP-ER) values (1.1 vs 3.5 and 2.8). In addition, and consistent with these relative AP-ER values, entrectinib (despite not reaching steady state) showed a more favourable CSF-to-plasma unbound concentration ratio than crizotinib and larotrectinib (both of which reached steady state) following constant intravenous infusion for $4-6 \mathrm{~h}$ in an in vivo brain distribution study ( $>0.2$ vs $\approx 0.03$ and $\approx 0.03$ ) [11]. Entrectinib demonstrated CNS penetration $[5,11]$ (e.g. steady-state brain/plasma ratios of 1.4-2.2, 0.6-1.0 and 0.4 following repeated daily oral administration in dog, rat and mouse, respectively [5]) and inhibited tumour growth and improved survival in murine models intracranially injected with human tumour cell lines driven by an NTRK [11] or $A L K$ [24] fusion.

\subsection{Resistance}

Experience with other TKIs suggests that acquired resistance to entrectinib limiting its effectiveness will inevitably emerge, either as a result of an 'on-target' mechanism (i.e. a target kinase domain mutation) or, alternatively, other, 'off-target' mechanisms (e.g. a bypass signalling pathway) [27-29]. Accordingly, reports of patients with NTRK+ solid tumours acquiring resistance to entrectinib have identified both 'on-target' mechanisms (e.g. G595R and G667C mutations in LMNA-NTRK1+ metastatic colorectal carcinoma [27], G595R mutation in TPR-NTRK1+ pancreatic cancer [29] and G623R mutation in ETV6-NTRK3+ MASC [30]) and an 'off-target' mechanism (emergent MET amplification in PLEKHA6-NTRK1+ cholangiocarcinoma [29]). Similarly, mechanisms of acquired resistance to entrectinib in $R O S 1+\mathrm{NSCLC}$ reportedly include the target kinase domain mutations G2032R, F2004C and F2004I [31] and-as identified in in vitro models using patient-derived tumour cells-activation of MET-[32] and RAS-[28] mediated bypass signalling.

Based on a blood analysis from an ongoing, global, phase II basket study (STARTRK-2; Sect. 4), acquired resistance mutations were detected in $10(34 \%)$ of the 29 evaluable patients in the NTRK+ solid tumour cohort and $5(28 \%)$ of 
the 18 evaluable patients in the ROS1+ NSCLC cohort, all of which were target kinase domain mutations. One additional patient from each cohort, both of whom had a partial response (PR) to entrectinib prior to progression, showed the emergence of a mutation in an oncogene within the MAPK pathway [31].

\section{Pharmacokinetic Properties of Entrectinib}

The pharmacokinetics of entrectinib in adult patients with solid tumours, like those of its major active metabolite, M5, are linear [20, 33] and not dose- or time-dependent [20]. Peak plasma concentrations of entrectinib and M5 were seen $\approx 4-6 \mathrm{~h}$ following single and multiple doses of oral entrectinib in the fed state; steady-state concentrations of entrectinib and M5 were reached within 2 weeks [34]. The estimated steady-state accumulation ratios for entrectinib and M5 were 1.89 and 2.01, respectively [20,33]. The bioavailability of entrectinib is not altered to clinically significant extent by food [18]. Both entrectinib and M5 are highly (> 99\%) bound to human plasma proteins [20]. The estimated apparent volumes of distribution for entrectinib and M5 are 551 and $81.1 \mathrm{~L}$, respectively [21].

Entrectinib is metabolized primarily by cytochrome P450 (CYP) 3A4 ( $\approx 76 \%$ ) to M5; it is also conjugated by uridine 5 '-diphospho-glucuronosyltransferase $1 \mathrm{~A} 4$ to its $\mathrm{N}$-glucuronide conjugate (M-11) [20]. Entrectinib is predominantly eliminated through hepatic metabolism; renal clearance has a minor role [ $83 \%$ of a radiolabelled oral dose was excreted in faeces (36\% as unchanged entrectinib; $22 \%$ as M5); $3 \%$ was excreted in urine] [20, 34]. The estimated apparent clearances of entrectinib and M5 are 19.6 and 52.4 $\mathrm{L} / \mathrm{h}$, respectively; the estimated elimination half-lives are 20 and $40 \mathrm{~h}$, respectively [20].

No clinically significant differences were seen in the pharmacokinetics of entrectinib, based on age (4-86 years), gender, bodyweight (32-130 kg), ethnicity (White, Asian and Black), mild hepatic impairment and mild to moderate renal impairment. The effects of moderate to severe hepatic impairment or severe renal impairment on pharmacokinetics of entrectinib are unknown [20]. In paediatric patients aged $\geq 12$ years, exposure to entrectinib following once-daily doses of $400 \mathrm{mg}$ [for body surface area (BSA) 1.11-1.50 $\mathrm{m}^{2}$ ) and $600 \mathrm{mg}$ (for BSA $\geq 1.51 \mathrm{~m}^{2}$ ) is predicted to be similar to that seen in adults following once-daily doses of $600 \mathrm{mg}$ [20].

Potential drug-drug interactions involving entrectinib, a weak P-gp substrate (Sect. 2) that is predominantly metabolized by CYP3A4, are summarized in Table 1 .

\section{Therapeutic Efficacy of Entrectinib}

The efficacy of entrectinib, as monotherapy, has been demonstrated in pooled cohorts of adults aged $\geq 18$ years with locally advanced (unresectable) or metastatic NTRK+ solid tumours or ROS1+ NSCLC, including those with CNS disease, who were treated in one of three, ongoing, openlabel, noncomparative, multicentre studies: two phase I trials (ALKA-372-001 and STARTRK-1); and one phase II global basket trial (STARTRK-2) [17, 20, 35-37]. All patients included in these prespecified integrated analyses had measurable disease per Response Evaluation Criteria in Solid Tumours (RECIST) version 1.1, an Eastern Cooperative Oncology Group performance status (ECOG PS) of $0-2$ and could have received previous cancer therapies, with the exception of TRK inhibitors (those with NTRK+ solid tumours) or ROS1 inhibitors (those with ROS1+ NSCLC) [17, 20, 35-37].

Eligible patients received entrectinib until disease progression or unacceptable toxicity; treatment could continue post-progression if the investigator decided that the patient continued to derive clinical benefit $[35,36]$. Initial (primary) analyses involving patients with non-CNS primary, NTRK+ solid tumours $(n=54)$ [35] or ROS1+ NSCLC $(n=53)$ [36] who received at least one dose of entrectinib $\geq 600$ $\mathrm{mg}$ once daily and who were followed up for a median of 12.9 and 15.5 months, respectively, have been published in full $[35,36]$. However, data discussed in this section are derived from updated analyses involving larger populations of patients with non-CNS primary NTRK+ solid tumours $(n=74)[20,37]($ Sect 4.1$)$ or ROS $1+\operatorname{NSCLC}(n=161)$ $[17,20]$ (Sect. 4.2) who received entrectinb $600 \mathrm{mg} /$ day and who were followed up for a median of 14.2 and 15.8 months, respectively. Results have been reported in full [17] and in the EU summary of product characteristics (SPC) [20], the European Public Assessment Report (EPAR) [23] and abstracts/posters/oral presentations [16, 37-44].

As in the initial analyses [35, 36], the primary endpoints in the updated analyses $[17,20,37]$ were objective response rate (ORR) and duration of response (DoR), as assessed by blinded independent central review (BICR) using RECIST v1.1. Secondary endpoints included IC ORR and DoR (by BICR) in patients with CNS metastases (asymptomatic or pretreated; measurable or non-measurable) at baseline per BICR [16, 17, 37]. Of note, time to CNS progression was assessed as a secondary and as an exploratory endpoint in patients with and without baseline CNS metastases per investigator, respectively. In this regard, patients with baseline CNS metastases per investigator underwent regular brain scans, whereas CNS follow-up of those without baseline CNS metastases per investigator was less 
Table 1 Summary of potential drug-drug interactions involving entrectinib [20]

\begin{tabular}{|c|c|}
\hline Drug(s) & Potential interaction \\
\hline \multirow[t]{2}{*}{$\begin{array}{l}\text { CYP3A and P-gp } \\
\text { inhibitors }\end{array}$} & $\begin{array}{l}\text { Itraconazole (strong CYP3A inhibitor) } \uparrow \text { ENT exposure by } 600 \% \text {; avoid coadministering ENT with moderate or strong } \\
\text { CYP3A inhibitors (e.g. ritonavir, itraconazole). If coadministration unavoidable, } \downarrow \text { ENT dose }\end{array}$ \\
\hline & $\begin{array}{l}\text { ENT is a weak P-gp substrate (in vitro); caution advised when coadministering it with moderate or strong P-gp inhibitors } \\
\text { (e.g. verapamil, felodipine, paroxetine) due to risk of } \uparrow \text { ENT exposure }\end{array}$ \\
\hline $\begin{array}{l}\text { CYP3A and P-gp } \\
\text { inducers }\end{array}$ & $\begin{array}{l}\text { Rifampin (strong inducer of both CYP3A and P-gp) } \uparrow \text { ENT exposure by } 77 \% \text {; avoid coadministering ENT with moderate } \\
\text { or strong CYP3A or P-gp inducers (e.g. carbamazepine, phenytoin, rifampin) }\end{array}$ \\
\hline \multirow[t]{2}{*}{$\begin{array}{l}\text { CYP3A and P-gp } \\
\text { substrates }\end{array}$} & $\begin{array}{l}\text { ENT is a weak CYP3A4 inhibitor; caution advised when coadministering it with sensitive CYP3A4 substrates that have a } \\
\text { narrow therapeutic range (e.g. cisapride, ergotamine, quinidine) due to } \uparrow \text { risk of adverse drug reactions }\end{array}$ \\
\hline & $\begin{array}{l}\text { ENT is a weak P-gp inhibitor. Its effect on coadministered digoxin (a sensitive P-gp substrate) is not clinically relevant, } \\
\text { but it is currently unknown whether its effect on more sensitive oral P-gp substrates (e.g. dabigatran etexilate) is larger }\end{array}$ \\
\hline $\begin{array}{l}\text { Other transporter } \\
\text { substrates }\end{array}$ & $\begin{array}{l}\text { ENT is a BCRP inhibitor and a weak OATP1B1 inhibitor (in vitro); caution advised when coadministering it with sensi- } \\
\text { tive oral BCRP substrates (e.g. methotrexate) or OATP1B1 substrates (e.g. atorvastatin) due to the risk of } \uparrow \text { absorption }\end{array}$ \\
\hline
\end{tabular}

$B C R P$ breast cancer resistance protein, $C Y P$ cytochrome $\mathrm{P} 450, E N T$ entrectinib, $O A T P$ organic anion transporting polypeptide, $P$ - $g p$ P-glycoprotein, $\uparrow$ increased, $\downarrow$ decreased

comprehensive, being based on symptomatic progression or routine CNS scans where customary $[16,17]$.

\subsection{NTRK+ Solid Tumours}

In the updated integrated analysis, the efficacy of entrectinib was assessed in 74 adult patients with NTRK+ solid tumours [37]. The median age of the cohort was 57 years; $52.7 \%$ of patients were female, $70.3 \%$ were Caucasian, $17.6 \%$ were Asian and $86.4 \%$ had an ECOG PS $\leq 1$ [37]. Nearly all patients had an NTRK1 $(n=30)$ or NTRK3 $(n=42)$ fusion [37]. Twelve different NTRK+ solid tumour types and > 25 histologies were represented, the most common cancers being sarcoma (21.6\%), NSCLC (17.6\%), MASC (17.6\%), colorectal cancer $(9.5 \%)$, thyroid cancer $(9.5 \%)$ and breast cancer $(8.1 \%)$ [37, 40]. The vast majority of patients $(97.3 \%)$ had metastatic disease, the most common sites being lung $(60.8 \%)$, lymph nodes $(52.7 \%)$ and brain $(25.7 \%$ per investigator $/ 21.6 \%$ per BICR) [20, 37].

Entrectinib induced clinically meaningful and durable responses in patients with advanced solid tumours harbouring NTRK fusions, demonstrating both systemic efficacy (irrespective of the presence or absence of CNS metastases at baseline) [37] and IC efficacy (in patients with CNS metastases at baseline) [16]. In this respect, systemic and IC efficacy findings in the subset of Asian patients with NTRK+ solid tumours $(n=13)$ were consistent with those in the overall study cohort $(n=74)$ [44].

In the overall cohort, the systemic ORR was $63.5 \%$ [comprising five complete responses (CRs) and $42 \mathrm{PRs}$ ] and the median DoR was 12.9 months [20, 37] (Table 2). Based on Kaplan-Meier (KM) estimates, the 6-, 9- and 12-month durable response rates (DRRs) were $71 \%, 65 \%$ and $55 \%$, respectively [20]. ORRs were consistent irrespective of baseline CNS disease status per BICR, although median
DoR, median progression-free survival (PFS) and median overall survival (OS) were shorter in patients with baseline CNS metastases than those without baseline CNS metastases [37] (Table 2), which is expected, as the former have a poorer prognosis (Sect. 1). Most responses were reported by the time of the first post-baseline tumour assessment at 1 month [37].

Entrectinib showed activity across multiple NTRK+ solid tumour types, including sarcoma (ORR of 56.3\%; $n=16$ ) [20], NSCLC [ORR of $69.2 \%$ (one CR and eight PRs); $n=13]$ [20, 41], MASC (ORR of $92.3 \% ; n=13$ ) [20] and various gastrointestinal cancers [ORR of 50.0\% (all PRs); $n=12$ ] [40]. Moreover, a beneficial effect was generally seen irrespective of prior therapy exposure (ORRs of $80.0 \%, 61.9 \%, 65.0 \%$ and $38.5 \%$ in patients who received $0,1,2$ and $\geq 3$ prior lines of systemic therapy in the metastatic setting, respectively) [39]. The relatively low ORR in the $\geq 3$ prior lines of systemic therapy subgroup may be a reflection of a poorer prognosis population (i.e. $31 \%$ of patients in this subgroup versus $0 \%, 19 \%$ and $10 \%$ in the 0,1 and 2 prior lines of systemic therapy subgroups, respectively, had an ECOG PS of 2) [39].

In terms of the CNS activity of entrectinib, the IC ORR in the subpopulation of eight patients with measurable baseline CNS metastases (including four who had received IC radiotherapy to the brain $<2$ months prior to starting entrectinib) was $62.5 \%$; the median IC DoR was not reached $[16,20]$ (Table 2). The IC ORR was also $62.5 \%$ (three CRs and two PRs) in the subpopulation of eight patients with NTRK+ NSCLC who had measurable or non-measurable baseline CNS metastases; it was $60 \%$ (three CR/PRs) in the subpopulation of five patients with $N T R K+$ NSCLC who had measurable baseline CNS metastases [38]. 
Table 2 Efficacy of oral entrectinib $600 \mathrm{mg}$ once daily in patients with NTRK fusion-positive solid tumours: pooled results from three ongoing phase I or II trials (ALKA-372-001; STARTRK-1 and STARTRK-2) [16, 37]

Pt population analysed (no. of pts)

Outcomes $^{\mathrm{a}}$

$\mathrm{ORR}^{\mathrm{b}}(\%) \quad \mathrm{CR}(\%) \quad \mathrm{PR}(\%) \quad \mathrm{DoR}^{\mathrm{b}}(\mathrm{mo})[95 \% \mathrm{CI}] \quad \mathrm{PFS}(\mathrm{mo})[95 \% \mathrm{CI}] \quad \mathrm{OS}(\mathrm{mo})[95 \% \mathrm{CI}]$

Systemic efficacy

\begin{tabular}{|c|c|c|c|c|c|c|}
\hline All pts (74) & 63.5 & 6.8 & 56.8 & $12.9[9.3-\mathrm{NE}]$ & $11.2[8.0-15.7]$ & $23.9[16.0-\mathrm{NE}]$ \\
\hline Pts with BL CNS metastases $^{c}(16)$ & 62.5 & 0 & 62.5 & $6.0[4.2-\mathrm{NE}]$ & $6.7[4.7-\mathrm{NE}]$ & $14.3[7.6-\mathrm{NE}]$ \\
\hline Pts without BL CNS metastases $^{\mathrm{c}}(58)$ & 63.8 & 8.6 & 55.2 & $12.9[9.3-\mathrm{NE}]$ & $12.0[8.7-16.0]$ & $23.9[16.8-\mathrm{NE}]$ \\
\hline \multicolumn{7}{|l|}{ IC efficacy ${ }^{\mathrm{d}}$} \\
\hline Pts with BL CNS metastases $^{\mathrm{c}}(16)$ & 50.0 & 25.0 & 25.0 & $8.0[6.7-\mathrm{NE}]$ & $8.9[5.9-14.3]$ & NR \\
\hline Pts with measurable $\mathrm{BL}^{\mathrm{c}} \mathrm{CNS}$ metastases (8) & 62.5 & 12.5 & 50.0 & $\mathrm{NE}[5.0-\mathrm{NE}]$ & $10.1[2.8-\mathrm{NE}]$ & NR \\
\hline
\end{tabular}

$B I C R$ blinded independent central review, $B L$ baseline, $C R$ complete response, $D o R$ duration of response, $I C$ intracranial, $N E$ not estimable, $N R$ not reported, $O R R$ objective response rate, $O S$ overall survival, $P F S$ progression-free survival, $P R$ partial response, $p t(s)$ patient(s), $R E C I S T$ Response Evaluation Criteria In Solid Tumours

${ }^{a}$ Evaluated by BICR using RECIST v1.1, except OS. DoR, PFS and OS values are medians

b(Co-)primary endpoint

${ }^{\mathrm{c}}$ Per BICR

${ }^{\mathrm{d}}$ Reported outcomes are IC-ORR (secondary endpoint), IC-CR, IC-PR, IC-DoR (secondary endpoint) and IC-PFS

Rates of detected CNS progression confirmed by scans were very low; the median time to CNS progression was not estimable at the time of the data cut-off (31 October 2018) in the overall cohort or the subpopulations of patients with and without baseline CNS metastases per investigator [16]. 3 (15.8\%) of the 19 patients with baseline CNS metastases per investigator experienced CNS progression, while none ( $0 \%)$ of the 55 patients without baseline CNS metastases per investigator developed new scan-confirmed brain lesions [16].

Patient-reported outcomes (PROs) were evaluated in 71 patients with NTRK+ solid tumours participating in the STARTRK-2 study [42]. At baseline, these patients reported moderate to high health-related quality of life (HR-QOL) and functioning, as assessed using the European Organisation for Research and Treatment of Cancer (EORTC) Quality of Life Questionnaire (QLQ-C30). While receiving entrectinib, they reported improvements in HR-QOL and physical and role functioning (including a clinically meaningful improvement in role functioning), as well as maintenance of cognitive function. They also reported a clinically meaningful improvement in pain. Patients with NTRK+ NSCLC $(n=12)$ also completed the EORTC Quality of Life Questionnaire-Lung Cancer Module (QLQ-LC13). They reported a moderate lung cancer symptom burden at baseline; their symptoms were unchanged or improved during treatment with entrectinib, including a clinically meaningful improvement in cough. Similarly, colorectal cancer-related symptoms, such as bloating, were unchanged or improved over time, as assessed in seven patients using the EORTC Quality of Life Questionnaire-Colorectal Cancer Module (QLQ-CR29) [42].

\subsubsection{Paediatric Patients}

The efficacy of entrectinib in paediatric patients aged $\geq 12$ years with $N T R K+$ solid tumours has been established on the basis of data extrapolation from adults with $N T R K+$ solid tumours enrolled in the ALKA-372-001, STARTRK-1 and STARTRK-2 studies (Sect. 4.1), and relevant data from children and adolescents aged $<21$ years with recurrent or refractory NTRK $+\mathrm{CNS} /$ solid tumours enrolled in another ongoing, open-label, non-comparative, multicentre, phase I/ II study (STARTRK-NG) [20, 45].

At the most recent data cut-off (5 November 2019) [45], 39 patients with relapsed or refractory CNS/solid tumours, with or without NTRK, ROS1 or ALK fusions, had been enrolled and evaluated in STARTRK-NG, including 14 with NTRK fusions. Best responses per investigator (Response Assessment in Neuro-Oncology criteria) in 11 patients with $N T R K+$ CNS primary tumours were four CRs and three PRs. Three patients with NTRK+ primary CNS tumours had stable disease; one had disease progression. Best responses per investigator in three patients with NTRK+ extracranial solid tumours were two CRs and one PR. All responses occurred at entrectinib doses of $\geq 400 \mathrm{mg} / \mathrm{m}^{2}$ [45].

\subsection{ROS1+ NSCLC}

In the updated integrated analysis, the efficacy of entrectinib was assessed in 161 adult patients with ROS1+ NSCLC [17]. The median age of the cohort was 54 years; $65 \%$ of patients were female, $44 \%$ were Caucasian, $45 \%$ 
were Asian, 37\% were current/former smokers and $90 \%$ had an ECOG PS $\leq 1$ [17]. The vast majority of patients (97.5\%) had adenocarcinoma; $98.1 \%$ had metastatic disease, with the most common sites being lymph nodes (69.6\%), lungs $(50.3 \%)$ and brain $(34.8 \%$ per investigator $28.6 \%$ per BICR) $[17,20]$.

Entrectinib therapy was associated with clinically meaningful and durable responses in patients with ROS1+ NSCLC, showing both systemic antitumour activity (irrespective of the presence or absence of baseline CNS metastases) and IC antitumour activity (in patients with baseline CNS metastases) [17]. In this respect, systemic and IC efficacy findings in the subset of Asian patients with ROS1+ NSCLC $(n=41)$ were consistent with those in the overall study cohort $(n=161)$ [44].

In the overall cohort, the systemic ORR was $67.1 \%$ (comprising $14 \mathrm{CRs}$ and $94 \mathrm{PRs}$ ) and the median DoR was 15.7 months [17] (Table 3). KM estimates of the 6-, 9- and 12-month DRRs were $83 \%, 75 \%$ and $63 \%$, respectively [17]. Median PFS, like median DoR, was 15.7 months; median OS was not estimable at the time of the data cut-off [17] (Table 3). The 6-, 9- and 12-month PFS rates were $77 \%$, $66 \%$ and $55 \%$, respectively; the 6-, 9- and 12-month OS rates were $91 \%, 86 \%$ and $81 \%$, respectively (KM estimates) [17].

ORRs were consistent irrespective of investigatorassessed CNS disease status at baseline, although median DoR and median PFS were, as expected, shorter in patients with baseline CNS metastases who have a poorer prognosis than those without baseline CNS metastases [17] (Table 3; Sect. 1). Median OS was not estimable at the time of the data cut-off in the overall cohort or the subpopulation of patients without baseline CNS metastases; it was 28.3 months in the subpopulation of patients with baseline CNS metastases [17] (Table 3).

Responses occurred early, with most reported at the first follow-up tumour assessment (1 month) [17]. Additionally, ORR benefit was observed to be generally irrespective of prior therapy exposure, being $71.7 \%, 60.9 \%, 66.7 \%$ and $73.7 \%$ in patients who received $0,1,2$ and $\geq 3$ prior lines of systemic therapy in the metastatic setting, respectively [39].

In terms of the CNS activity of entrectinib, the IC ORR in the subpopulation of 24 patients with measurable baseline CNS metastases (including nine who had received IC radiotherapy to the brain $<2$ months before initiating entrectinib) was $79.2 \%$; the median IC DoR was 12.9 months (Table 3). KM estimates of the 6-, 9- and 12-month IC DRRs were $77 \%, 61 \%$ and $55 \%$, respectively [17]. The median time to radiologically-confirmed CNS progression was not estimable at the time of the data cut-off (1 May 2019) in the overall cohort or the subpopulation of patients without baseline CNS metastases per investigator; it was 13.6 months in the subpopulation of patients with baseline CNS metastases per investigator [38]. Twenty-seven (48.2\%) of the 56 patients with baseline CNS metastases per investigator experienced CNS progression, while new scan-confirmed brain lesions were discovered in $3(2.9 \%)$ of the 105 patients without baseline CNS metastases per investigator [17].

PROs were evaluated in 145 patients with ROS1 + NSCLC participating in the STARTRK-2 study [43]. At baseline, they reported moderate to high HR-QOL and functioning (assessed using the QLQ-C30); while receiving entrectinib, they reported non-clinically meaningful improvements in

Table 3 Efficacy of oral entrectinib $600 \mathrm{mg}$ once daily in patients with ROS1 fusion-positive metastatic NSCLC: pooled results from three ongoing phase I or II trials (ALKA-372-001; STARTRK-1 and STARTRK-2) [17]

Pt population analyzed (no. of pts)

Outcomes $^{\mathrm{a}}$

$\mathrm{ORR}^{\mathrm{b}}(\%) \quad \mathrm{CR}(\%) \quad \mathrm{PR}(\%) \quad \mathrm{DoR}^{\mathrm{b}}(\mathrm{mo})[95 \% \mathrm{CI}] \quad \mathrm{PFS}(\mathrm{mo})[95 \% \mathrm{CI}] \quad \mathrm{OS}(\mathrm{mo})[95 \% \mathrm{CI}]$

\begin{tabular}{lllllll} 
Systemic efficacy & & & & & \\
\hline All pts (161) & 67.1 & 8.7 & 58.4 & $15.7[13.9-28.6]$ & $15.7[11.0-21.1]$ & NE [28.3-NE] \\
\hline Pts with BL CNS metastases $^{\mathrm{c}}(56)$ & 62.5 & 7.1 & 55.4 & $14.9[9.6-20.5]$ & $11.8[6.4-15.7]$ & $28.3[16.1-\mathrm{NE}]$ \\
\hline Pts without BL CNS metastases $^{\mathrm{c}}(105)$ & 69.5 & 9.5 & 60.0 & $24.6[13.9-34.8]$ & $19.0[12.0-29.6]$ & $\mathrm{NE}[30.8-\mathrm{NE}]$ \\
\hline${\text { IC } \text { efficacy }^{\mathrm{d}}}^{\text {Pts with BL CNS metastases }^{\mathrm{e}}(46)}$ & & & & & & \\
\hline Pts with measurable BL CNS metastases $^{\mathrm{e}}(24)$ & 79.2 & 12.5 & 66.7 & $12.9[6.8-22.1]$ & $12.0[6.2-19.3]$ & NR \\
\hline
\end{tabular}

$B I C R$ blinded independent central review, $B L$ baseline, $C R$ complete response, $D o R$ duration of response, $I C$ intracranial, $N E$ not estimable, $N R$ not reported, $O R R$ objective response rate, $O S$ overall survival, $P F S$ progression-free survival, $p t(s)$ patient(s), $P R$ partial response, $R E C I S T$ Response Evaluation Criteria In Solid Tumours

${ }^{a}$ Evaluated by BICR using RECIST v1.1, except OS. DoR, PFS and OS values are medians

b(Co-)primary endpoint

${ }^{c}$ Per investigator

${ }^{\mathrm{d}}$ Reported outcomes are IC-ORR (secondary endpoint), IC-CR, IC-PR, IC-DoR (secondary endpoint) and IC-PFS

${ }^{\mathrm{e}}$ Per BICR 
HR-QOL and physical and role functioning scores that were accompanied by a non-clinically meaningful reduction in the cognitive functioning score. In general, tumour-related symptoms (assessed using the QLQ-LC13) were unchanged or improved during treatment with entrectinib, including a clinically meaningful improvement in cough [43].

\section{Tolerability of Entrectinib}

Entrectinib was generally well tolerated, based on initial $[35,36]$ and updated $[20,23]$ analyses of pooled safety data from the four ongoing phase I/II trials (ALKA-372-001 and STARTRK-1, -2 and -NG).

The integrated safety population comprised 504 patients (including 475 adults) who had received at least one dose of entrectinib; they had been exposed to the drug for a median of 5.5 months [20, 23]. The most common adverse drug reactions (ADRs) were fatigue (in $45 \%$ of patients), constipation (42.9\%), dysgeusia (42.3\%), dizziness (39.7\%), oedema (37.3\%), diarrhoea (33.5\%), nausea (32.1\%), dysaesthesia (29.0\%), anaemia (28.2\%), dyspnoea (27.0\%), weight increased (26.4\%), blood creatinine increased (25.4\%), pain (24.4\%), cognitive disorders [24.2\% (29.7\% and $23.1 \%$ in patients with and without baseline CNS disease, respectively)], vomiting (23.2\%), cough (21.4\%) and pyrexia (20.0\%) [20]. Cognitive disorders included events reported as cognitive disorders $(6.3 \%)$, confusional state $(7.3 \%)$, memory impairment (4.2\%), attention disturbance (3.8\%), amnesia (2.8\%), mental status changes (1.2\%), hallucination $(1.0 \%)$, delirium $(0.8 \%)$, visual hallucination $(0.4 \%)$ and mental disorder $(0.2 \%)$ [20].

The most common grade $\geq 3$ ADRs were anaemia $(9.7 \%)$, weight increased (7.3\%), lung infection (6.0\%), dyspnoea (5.8\%) and fatigue (5.0\%); the most frequent serious ADRs were lung infection (5.2\%), dyspnoea (4.6\%), cognitive impairment (3.8\%) and pleural effusion (2.4\%) [20]. Most ADRs were transient and managed successfully with dose interruption or reduction [35, 36]; the rate of permanent entrectinib discontinuation due to ADRs was low (4.4\%) [20].

The overall safety profile of entrectinib was similar in patients with NTRK+ solid tumours and those with ROS1+ NSCLC $[35,36]$. Likewise, it was similar in elderly patients aged $\geq 65$ years $(n=130)$ and younger patients aged $<65$ years, albeit dizziness ( $48.5 \%$ vs $36.6 \%$ ), blood creatinine increased $(31.5 \%$ vs $23.3 \%)$, ataxia $(23.8 \%$ vs $12.8 \%)$ and hypotension ( $21.5 \%$ vs $14.7 \%$ ) occurred more frequently in the former than the latter [20].

The overall safety profile of entrectinib also appeared to be similar in paediatric patients and adults, based on updated data for 32 paediatric patients (30 enrolled in STARTRKNG; two enrolled in STARTRK-2), including seven adolescents aged 12-17 years [20]. Grade 3 or 4 ADRs/laboratory abnormalities that occurred with an incidence $\geq 5 \%$ higher in paediatric than adult patients were neutropenia ( $28.1 \%$ vs $3.4 \%)$, weight increased $(21.9 \%$ vs $6.9 \%)$, headache $(6.3 \%$ vs $0.6 \%)$ and bone fractures $(12.5 \%$ vs $1.9 \%$ ) [20]. Grade $\geq$ 3 ADRs reported in adolescents were neutropenia and headache (no further details are available) [20].

Fractures were experienced by 25 (5.3\%) of the 475 adult patients and seven $(21.8 \%)$ of the 32 paediatric patients [20]. Most fractures were hip or other lower extremity fractures (e.g. femoral or tibial shaft); the median time to fracture was 3.4 and 4.3 months in adults and paediatric patients, respectively. The seven paediatric patients were all aged $<12$ years; they reported a total of 11 ADRs of fractures, including four grade 3 events (of which three were serious). No patient discontinued entrectinib due to fractures, and all but one event of fracture (in a paediatric patient) recovered. Some fractures in adults occurred in the setting of a fall or other trauma to the affected area, and there were also some reports of possible tumour involvement at the site of fracture. In contrast, all fractures in paediatric patients occurred in association with minimal or no trauma, and there were no reports of tumour involvement at the site of the fracture [20].

Other notable ADRs occurring in entrectinib recipients $(n$ $=504$ ) included peripheral sensory neuropathy (in $15.7 \%$ of patients), hyperuricaemia (in $9.1 \%$ of patients; grade $\geq 3$ in $1.8 \%)$, congestive heart failure [(CHF) in $3.0 \%$ of patients; grade $\geq 3$ in $2.2 \%$ ], syncope (in $4.6 \%$ of patients; concurrently with hypotension, dehydration or QTc prolongation in some individuals), eye disorders [e.g. vision blurred (in $8.5 \%$ of patients), diplopia (2.6\%) and visual impairment (1.6\%)] and ECG QTc prolongation (in $2.0 \%$ of patients) [20]. Among patients with $\geq 1$ post-baseline ECG assessment, $17(4.0 \%)$ had a Fridericia QTc prolongation of $>60$ ms; 12 (2.8\%) had a Fridericia QTc of $\geq 500 \mathrm{~ms}$ ] [20].

\section{Dosage and Administration of Entrectinib}

In the EU, entrectinib has been conditionally approved for the treatment of adult and paediatric patients aged $\geq 12$ years with $N T R K+$ solid tumours who have a disease that is locally advanced, metastatic or where surgical resection is likely to result in severe morbidity; have not received a prior TRK inhibitor; and have no satisfactory treatment options. It has also been conditionally approved as monotherapy for the treatment of adult patients with advanced $R O S 1+$ NSCLC not previously treated with ROS1 inhibitors [20].

Similarly, in the USA, entrectinib has been conditionally approved for the treatment of adult and paediatric patients aged $\geq 12$ years with solid tumours that have a NTRK gene fusion without a known acquired resistance mechanism; are metastatic or where surgical resection is likely to result in severe morbidity; and have progressed following treatment 
or have no satisfactory alternative therapy. It has also been conditionally approved for the treatment of adult patients with metastatic NSCLC whose tumours are ROS1+ [21].

The recommended dosage in adults with ROS1+ NSCLC or NTRK+ solid tumours is $600 \mathrm{mg}$ once daily. The recommended once-daily dosage in paediatric patients aged $\geq 12$ years with $N T R K+$ solid tumours is based on body surface area (see local prescribing information for details) [20, 21]. Entrectinib may be taken with or without food; however, it should not be administered with grapefruit/juice (Sect. 3). It is recommended that treatment be continued until disease progression or unacceptable toxicity [20, 21].

Local prescribing information should be consulted for detailed information regarding dose modifications to manage ADRs, potential drug interactions, use in special patient populations, contraindications, and other warnings and precautions.

\section{Place of Entrectinib in the Management of NTRK+ Solid Tumours and ROS1+ NSCLC}

Entrectinib is an orally active, CNS-penetrant, small-molecule TKI of TRKA/B/C, ROS 1 and ALK that has been developed to fulfill unmet clinical needs, including for a targeted therapy in NTRK+ solid tumours and ROS1 + NSCLC that has activity against brain tumours (either primary or metastatic) in addition to systemic efficacy (Sect. 1).

The efficacy of entrectinib monotherapy in treating a variety of locally-advanced or metastatic $N T R K+$ solid tumours in TRK inhibitor-naïve adults has been established according to an integrated analysis of three ongoing, single-arm trials, in which the drug induced clinically meaningful and durable systemic responses independent of the tumour type as well as the NTRK fusion type, and irrespective of the presence or absence of CNS metastases at baseline (Sect. 4.1). Moreover, in patients with measurable CNS metastases at baseline, the IC efficacy of entrectinib was substantial and comparable to its systemic efficacy (Sect. 4.1). The efficacy of entrectinib in paediatric patients aged $\geq 12$ years with $N T R K+$ solid tumours was established, based on extrapolation of data from the single-arm studies in adults and relevant data from children and adolescents with recurrent or refractory NTRK+ CNS/solid tumours enrolled in another single-arm study (Sect. 4.1.1). Patients aged $<21$ years with recurrent or refractory solid tumours (including primary CNS tumours) harbouring NTRK or ROS1 fusions are continuing to be enrolled in the expansion cohorts of this ongoing trial [45].

Due to its demonstrable activity against solid tumours harbouring a specific molecular alteration (in this case an NTRK fusion), regardless of their location, entrectinib is referred to as a 'tumour-agnostic' or 'histology-independent' therapy. It is the second such tumour-agnostic therapy to be conditionally approved for the treatment of locally-advanced or metastatic $N T R K+$ solid tumours, after larotrectinib (also on the basis of an integrated analysis of efficacy in three single-arm trials [46]). In terms of the EU-approved indication, the main difference between the two TKIs is that entrectinib is indicated for adults and paediatric patients aged $\geq 12$ years [20], whereas larotrectinib is indicated for adults and paediatric patients of all ages [46]. Additionally, the EU label for entrectinib [20], unlike that for larotrectinib [46], specifies that patients must not have received a prior TRK inhibitor (Sect. 6). The conditional approval status of entrectinib and larotrectinib in the EU means that further evidence on these medicinal products is awaited; the European Medicines Agency will review new information at least annually and update the SPC as necessary [20, 46].

Entrectinib, like larotrectinib, has been incorporated into National Comprehensive Cancer Network (NCCN) guidelines pertaining to the treatment of various advanced or metastatic NTRK+ solid tumours [47] (Table 4). For example, both TKIs are recommended as a preferred option for the first-line treatment of patients with advanced or metastatic NTRK+ NSCLC; they are also recommended as an option for subsequent therapy in these patients following progression on alternative first-line systemic therapies that may be useful in certain circumstances (e.g. albumin-bound paclitaxel, docetaxel and gemcitabine) [47] (Table 4). With respect to tumours in the CNS, both entrectinib and larotrectinb are recommended as a systemic therapy for $N T R K+$ solid tumours with brain metastases [47] (Table 4). In this regard, entrectinib has demonstrable IC efficacy in adults with $N T R K+$ solid tumours and brain metastases (Sect. 4.1). Furthermore, there are data (albeit with limitations and therefore requiring cautious interpretation) suggestive of a potential CNS-protective effect of entrectinib in terms of preventing the onset of new lesions in patients without baseline CNS metastases (Sect. 4.1). Larotrectinib has also reportedly shown IC activity, including in a small number of patients with $N T R K+$ solid tumours and measurable brain metastases at baseline [48]. However, due to the rarity of $N T R K+$ solid tumours, directly comparing the two TKIs in randomized trials (e.g. focusing on IC activity in patients with metastatic brain tumours) is not considered feasible. Moreover, any indirect comparison of the two TKIs based on their existing integrated analyses of efficacy will be subject to a number of limitations. These include reliance on data derived from single-arm trials, low patient numbers and heterogeneous study populations, notably with respect to age (i.e. the larotectinib, but not entrectinib, cohort includes both adults and paediatric patients), the proportions of patients with primary/metastatic brain tumours (e.g. the entrectinib cohort currently 
has a higher proportion of patients with CNS metastases than the larotrectinib cohort $[20,46,49])$, the types and relative proportions of the NTRK+ solid tumours represented and the number and type of prior lines of therapy. Further expansion of the integrated analyses in addition to the emergence of real-world data from routine clinical practice will increase the evidence base for, and hence the robustness of, indirect comparisons of the relative efficacy of these two agents [7]; hence, both developments are awaited with interest. On-target resistant mutations have been identified in patients who have progressed on entrectinib (Sect. 2.1), as well as larotrectinib, indicating that these represent a shared liability for these first-generation TRK inhibitors [49]. Second-generation TRK inhibitors designed to overcome acquired on-target resistance to firstgeneration agents are currently being evaluated $[49,50]$.

The efficacy of entrectinib monotherapy in treating in ROS1 + NSCLC in ROS1 inhibitor-naïve adults has also been established according to the integrated analysis of the three ongoing, single-arm trials, in which the drug induced clinically meaningful and durable systemic responses, both in patients with and without baseline CNS metastases (Sect. 4.2). Entrectinib showed marked IC antitumour activity in patients with baseline CNS metastases and, as for patients with NTRK+ solid tumours, there are data (albeit with limitations and hence requiring cautious interpretation) suggestive of a potential CNS-protective effect of entrectinib in patients without baseline CNS metastases (Sect. 4.2).

Importantly, the efficient brain penetration and potent IC activity of entrectinib represents a potential advantage over crizotinib, the current standard of care for ROS1+ NSCLC, which demonstrates poor brain penetration and, hence, limited CNS activity [51] (Sect. 1). Accordingly, as set out in the NCCN guideline for ROS1 + NSCLC [47], entrectinib is a preferred first-line therapy alongside crizotinib, albeit with a note that it may be better for patients with brain metastases.

A head-to-head study directly comparing entrectinib with crizotinib in patients with advanced ROSI+ NSCLC, with or without CNS metastases, has commenced (NCT04603807). This randomized, open-label, multicentre, phase III study

Table 4 Recommendations regarding entrectinib as a treatment option in patients with NTRK fusion-positive solid tumours: summary of National Comprehensive Cancer Network (NCCN) guidelines [47]

\begin{tabular}{|c|c|}
\hline Tumour type & Recommendation $^{\mathrm{a}}$ \\
\hline Breast cancer & Option in recurrent/stage IV disease in pts with no satisfactory alternatives or who have progressed after treatment \\
\hline Cervical cancer & Option for 2nd-line treatment of recurrent/metastatic disease \\
\hline Colon/rectal cancer & Subsequent-line option for metastatic disease following initial therapy \\
\hline Cutaneous melanoma & Option for 2nd-line or subsequent-line treatment of metastatic or unresectable disease \\
\hline CNS cancers & Option for tumours with newly-diagnosed or recurrent brain metastases \\
\hline Gastric cancer & $\begin{array}{l}\text { Other recommended regimens option for } 2 \text { nd-line or subsequent-line treatment of unresectable locally advanced, } \\
\text { recurrent, or metastatic disease, where local therapy is not indicated }\end{array}$ \\
\hline Head/neck cancers & Option for treatment of recurrent salivary gland tumours with distant metastases in pts with a PS of $0-3$ \\
\hline Hepatobiliary cancers & $\begin{array}{l}\text { Subsequent-line option for HCC that is: unresectable and pt is not a transplant candidate } \text { or inoperable by PS } \\
\text { or comorbidity, local/local with minimal extrahepatic disease only or metastatic or has extensive liver tumour } \\
\text { burden } \\
\text { Option for primary treatment and subsequent-line therapy upon disease progression of unresectable or metastatic } \\
\text { gallbladder cancer, ICC or ECC }\end{array}$ \\
\hline NSCLC & Preferred option for 1st-line and option for subsequent-line treatment of pts with advanced or metastatic disease \\
\hline Oesophageal cancer/EJCs & $\begin{array}{l}\text { Other recommended regimens option for } 2 \text { nd-line or subsequent-line treatment of unresectable locally advanced, } \\
\text { recurrent, or metastatic oesophageal cancer or EJCs, where local therapy is not indicated }\end{array}$ \\
\hline Ovarian cancer & $\begin{array}{l}\text { Option as recurrence therapy for platinum-sensitive and -resistant disease in pts with EOC, FTC, PPC, including } \\
\text { LCOH }\end{array}$ \\
\hline Pancreatic adenocarcinoma & $\begin{array}{l}\text { 1st-line option for metastatic disease in pts with poor PS; 2nd-line option for locally advanced/metastatic disease or } \\
\text { therapy for recurrent disease in pts with good or poor PS }\end{array}$ \\
\hline STS & Option for STS subtypes with non-specific histologies (not intended for adjuvant therapy of non-metastatic disease) \\
\hline Thyroid cancer & $\begin{array}{l}\text { Option for treatment of advanced, progressive, or threatening papillary carcinoma, follicular carcinoma, or Hurthle } \\
\text { cell carcinoma; preferred regimen for treatment of metastatic (stage IVC) anaplastic carcinoma }\end{array}$ \\
\hline Vulvar cancer & Option for treatment of advanced, recurrent or metastatic disease \\
\hline
\end{tabular}

$E C C$ extrahepatic cholangiocarcinoma, EJCs esophagogastric junction cancers, EOC epithelial ovarian cancer, FTC fallopian tube cancer, $H C C$ hepatocellular carcinoma, ICC intrahepatic cholangiocarcinoma, $L C O H$ less common ovarian cancers, NSCLC non-small cell lung cancer, $P P C$ primary peritoneal cancer, $p t(s)$ patient(s), $P S$ performance status, $S T S$ soft tissue sarcoma

${ }^{a}$ Also applies to larotrectinib 
will enrol an estimated 220 patients and has an estimated completion date of late 2027; the primary endpoint is PFS in participants with baseline CNS metastases. Currently, the relative benefits of these two TKIs have been comprehensively assessed using the matching-adjusted indirect comparisons method [52]. Briefly, data for entrectinib were derived from the initial integrated analysis of the ALKA372-001, STARTRK-1 and STARTRK-2 trials, while that for crizotinib were derived from the pivotal PROFILE 1001 study. Matching was based on known prognostic/predictive factors and, notably, the proportion of patients with CNS metastases (not reported in PROFILE 1001) was varied across three scenarios. The results of this analysis suggested improved outcomes with entrectinib versus crizotinib, with the former yielding significantly better ORRs than the latter in all three scenarios. OS and treatment discontinuation due to adverse events also favoured entrectinib, while PFS was generally similar [52]. Another analysis that indirectly compared the entrectinib clinical trial population (initial integrated analysis) with a matched real-world crizotinib population also suggested improved outcomes with the former versus the latter in terms of a longer PFS and a longer time to treatment discontinuation [53]. Accordingly, data on the effectiveness of entrectinib in ROS1+ NSCLC in clinical practice are awaited with interest, as are comparisons of the drug with crizotinib, based on real-world cohorts of ROS1+ NSCLC patients.

The NCCN guideline for ROS1+ NSCLC [47] also recommends entrectinib as an option for subsequent therapy, albeit with a note that it is primarily for patients with CNS progression after crizotinib [47]. The extent of the role of entrectinib in patients who develop systemic disease progression due to crizotinib resistance is uncertain [54], primarily because it does not show activity against the most common acquired mutation that confers resistance to crizotinib in ROS1+ NSCLC, namely ROS1-G2032R [15] (Sect. 2.1). The administration of entrectinib to ROS1 + NSCLC patients with acquired resistance to crizotinib is not supported by the limited amount of relevant data currently available [54]; further reports of experience with the drug in this setting would be of considerable interest. Most crizotinib-treated patients eventually progress, either as a result of acquired resistance or, frequently, the development of CNS metastases; this, in view of the CNS activity of entrectinib and its ineffectiveness against ROS1-G2032R, argues in favour of using entrectinib before, rather than after, crizotinib in advanced ROS1+ NSCLC, particularly in patients with baseline brain metastases $[17,54]$. ROS1 TKIs with broad activity against kinase mutations, including ROS1-G2032R, are currently being evaluated $[50,54]$.
Of note, PROs from patients with $N T R K+$ solid tumours or ROS1 + NSCLC enrolled in STARTRK-2 have been presented in preliminary form (Sect. 4). The results support a positive benefit-risk profile for entrectinib; importantly, the drug is associated with a minimal treatment-related symptom burden [42, 43].

Entrectinib was generally well tolerated, with a manageable safety profile that was similar in patients with NTRK+ solid tumours and those with ROS1+ NSCLC, as well as in paediatric patients, younger adults and the elderly, based on data pooled from two or all four of the ongoing, single arm trials (Sect. 5). Among the most commonly reported ADRs, dizziness, weight increased and cognitive disorders are potentially related to TRK inhibition; hence, their occurrence is not unexpected [36]. Local prescribing information (e.g. the EU SPC [20]) should be consulted for recommended entrectinib dose modifications (interruption, reduction or discontinuation) to manage ADRs, such as cognitive changes, hyperuricaemia, QT interval prolongation and CHF (Sect. 6). According to the EU SPC [20], entrectinib should be avoided in patients with a baseline corrected QT interval (QTc) > $450 \mathrm{~ms}$, those with congenital long QTc syndrome, and those taking medicinal products known to prolong the QTc interval [20]. Entrectinib should also be avoided in patients with electrolyte imbalances or significant cardiac disease, including recent myocardial infarction, $\mathrm{CHF}$, unstable angina and bradyarrhythmias. Additional monitoring should be performed (e.g. assessment of left ventricular ejection fraction in patients with symptoms or known risk factors of CHF) and a specialist consultation considered, if the drug is to be prescribed to patients with any of these conditions. Assessment of ECG and electrolytes at baseline, 1 month after starting entrectinib and periodically throughout treatment (as clinically indicated) is recommended [20].

The assays currently required to select patients with $N T R K+$ solid tumours or ROS1+ NSCLC who are eligible to receive entrectinib are typically time-consuming and rely on invasive tissue biopsies [55]. Hence, a non-invasive (in vitro), next-generation sequencing-based companion diagnostic [FoundationOne ${ }^{\circledR}$ Liquid CDx $\left(\mathrm{F} 1 \mathrm{~L}^{\circledR} \mathrm{CDx}\right)$ ] is under development [56]. Recently, the clinical validity of the $\mathrm{F} 1 \mathrm{~L}^{\circledR} \mathrm{CDx}$ assay as a complementary aid to tissue-based testing for the identification of patients with $N T R K+$ solid tumours and ROS1+ NSCLC who may benefit from entrectinib treatment was demonstrated in a bridging study [55].

In conclusion, entrectinib expands the range of treatment options for advanced NTRK+ solid tumours and ROSI+ NSCLC, and may be of particular value in patients with existing CNS metastases and those who are at risk of developing CNS metastases. 


\section{Data Selection Entrectinib: 293 records identified}

Duplicates removed

Excluded during initial screening (e.g. press releases; news reports; not relevant drug/indication; preclinical study; reviews; case reports; not randomized trial)

Excluded during writing (e.g. reviews; duplicate data; small patient number; nonrandomized/phase I/II trials)

Cited efficacy/tolerability articles Cited articles not efficacy/tolerability 11 45

Search Strategy: EMBASE, MEDLINE and PubMed from 1946 to present. Clinical trial registries/databases and websites were also searched for relevant data. Key words were entrectinib, Rozlytrek, NSCLC, solid tumours. Records were limited to those in English language. Searches last updated 25 March 2021.

Electronic supplementary material The online version of this article (https://doi.org/10.1007/s40265-021-01503-3) contains supplementary material, which is available to authorized users.

Acknowledgements During the peer review process, the manufacturer of entrectinib was also offered an opportunity to review this article. Changes resulting from comments received were made on the basis of scientifc and editorial merit.

\section{Declarations}

Funding The preparation of this review was not supported by any external funding.

Authorship and Conflict of interest J. E. Frampton is a salaried employee of Adis International Ltd/Springer Nature, and declares no relevant conflicts of interest. All authors contributed to the review and are responsible for the article content.

Ethics approval, Consent to participate, Consent to publish, Availability of data and material, Code availability Not applicable.

Open Access This article is licensed under a Creative Commons Attribution-NonCommercial 4.0 International License, which permits any non-commercial use, sharing, adaptation, distribution and reproduction in any medium or format, as long as you give appropriate credit to the original author(s) and the source, provide a link to the Creative Commons licence, and indicate if changes were made. The images or other third party material in this article are included in the article's Creative Commons licence, unless indicated otherwise in a credit line to the material. If material is not included in the article's Creative Commons licence and your intended use is not permitted by statutory regulation or exceeds the permitted use, you will need to obtain permission directly from the copyright holder. To view a copy of this licence, visit http://creativecommons.org/licenses/by-nc/4.0/.

\section{References}

1. Jürgensmeier JM, Eder JP, Herbst RS. New strategies in personalized medicine for solid tumors: molecular markers and clinical trial designs. Clin Cancer Res. 2014;20(17):4425-35.
2. Cocco E, Scaltriti M, Drilon A. NTRK fusion-positive cancers and TRK inhibitor therapy. Nat Rev Clinl Oncol. 2018;15(12):731-47.

3. Awad K, Dalby M, Cree I, et al. The precision medicine approach to cancer therapy: part 1 - solid tumours. Pharm J. 2019. https:// doi.org/10.1211/PJ.2019.20207119.

4. El-Diery WS, Goldberg RM, Lenz H-J, et al. The current state of molecular testing in the treatment of patients with solid tumors, 2019. CA Cancer J Clin. 2019;69(4):305-43.

5. Rangaraju S, Farago A, Heym KM, et al. Preclinical and clinical efficacy of entrectinib in primary and metastatic brain tumors harboring NTRK, ROS1, or ALK gene fusions [abstract no.P14.19]. Neuro Oncol. 2017;19(Suppl 3):iii106.

6. Gatalica Z, Xiu J, Swensen J, et al. Molecular characterization of cancers with NTRK gene fusions. Mod Pathol. 2019;32:2147-53.

7. Chu P, Batson S, Hodgson M, et al. Systematic review of neurotrophic tropomyosin-related kinase inhibition as a tumor-agnostic management strategy. Future Oncol. 2020;16(4):61-74.

8. Farago AF, Taylor MS, Zhu VW, et al. Clinicopathologic features of non-small-cell lung cancer harboring an NTRK gene fusion. JCO Precis Oncol. 2018;2018:PO.18.00037.

9. Laetsch TW, Hawkins DS. Larotrectinib for the treatment of TRK fusion solid tumors. Expert Rev Anticancer Ther. 2019;19(1):1-10.

10. Frampton JE. Crizotinib: a review of its use in the treatment of anaplastic lymphoma kinase-positive, advanced non-small cell lung cancer. Drugs. 2013;73:2031-51.

11. Fischer H, Ullah M, de la Cruz CC, et al. Entrectinib, a TRK/ ROS1 inhibitor with anti-CNS tumor activity: differentiation from other inhibitors in its class due to weak interaction with P-glycoprotein. Neuro Oncol. 2020;22(6):819-29.

12. Soffietti R, Ahluwalia M, Lin N, et al. Management of brain metastases according to molecular subtypes. Nat Rev Neurol. 2020;16(10):557-74.

13. Farago AF, Taylor MS, Doebele RC, et al. Clinicopathologic features of non-small-cell lung cancer harboring an NTRK gene fusion. JCO Precis Oncol. 2018;2:PO.18.00037.

14. Patil T, Smith DE, Bunn PA, et al. The incidence of brain metastases in stage IV ROS1-rearranged non-small cell lung cancer and rate of central nervous system progression on crizotinib. J Thorac Oncol. 2018;13:1717-26.

15. Gainor JF, Tseng D, Yoda S, et al. Patterns of metastatic spread and mechanisms of resistance to crizotinib in ROS1positive non-small-cell lung cancer. JCO Precis Oncol. 2017;2017:PO.17.00063.

16. John T, Chiu CH, Cho B, et al. Intracranial efficacy of entrectinib in patients with NTRK fusion-positive solid tumours and baseline CNS metastases [abstract no. 3640 plus oral presentation]. In: ESMO Virtual Congress; 2020.

17. Dziadziuszko R, Krebs MG, De Braud F, et al. Updated integrated analysis of the efficacy and safety of entrectinib in locally advanced or metastatic ROS1 fusion-positive non-small-cell lung cancer. J Clin Oncol. 2021. https://doi.org/10.1200/JCO.20.03025.

18. Menichincheri M, Ardini E, Magnaghi P, et al. Discovery of entrectinib: a new 3-aminoindazole as a potent anaplastic lymphoma kinase (ALK), c-ros oncogene 1 kinase (ROS1), and pantropomyosin receptor kinases (Pan-TRKs) inhibitor. J Med Chem. 2016;59(7):3392-408.

19. Liu D, Offin M, Harnicar S, et al. Entrectinib: an orally available, selective tyrosine kinase inhibitor for the treatment of NTRK, $R O S 1$, and $A L K$ fusion-positive solid tumors. Ther Clin Risk Manag. 2018;14:1247-52.

20. European Medicines Agency. Rozlytrek 100 and $200 \mathrm{mg}$ hard capsules: EU summary of product characteristics. 2020. https:// www.ema.europa.eu. Accessed 25 Mar 2021. 
21. Genentech. ROZLYTREK (entrectinib) capsules, for oral use: US prescribing information. 2019. https://www.accessdata.fda.gov/ cder. Accessed 25 Mar 2021.

22. Chugai Pharmaceutical Co. Ltd. Rozlytrek (entrectinib) capsules: Japanese prescribing information. 2019. http://www.pmda.go.jp. Accessed 25 Mar 2021.

23. European Medicines Agency. CHMP assessment report: Rozlytrek: international non-proprietary name: entrectinib: procedure no. EMEA/H/C/004936/0000. 2020. https://www.ema. europa.eu. Accessed 25 Mar 2021.

24. Ardini E, Menichincheri M, Banfi P, et al. Entrectinib, a pan-TRK, ROS1, and ALK inhibitor with activity in multiple molecularly defined cancer indications. Mol Cancer Ther. 2016;15(4):628-39.

25. Smith KM, Fagan PC, Pomari E, et al. Antitumor activity of entrectinib, a Pan-TRK, ROS1, and ALK Inhibitor, in ETV6NTRK3-positive acute myeloid leukemia. Mol Cancer Ther. 2018;17(2):455-63.

26. Wei G, Ardini E, Patel R, et al. Entrectinib is effective against the gatekeeper and other emerging resistance mutations in NTRK-, ROS1-and $A L K$-rearranged cancers [abstract no. 2136]. Cancer Res. 2016;76(14 Suppl).

27. Russo M, Misale S, Wei G, et al. Acquired resistance to the TRK inhibitor entrectinib in colorectal cancer. Cancer Discov. 2016;6(1):36-44.

28. $\mathrm{Ku} \mathrm{BM}, \mathrm{Bae} \mathrm{YH}$, Lee KY, et al. Entrectinib resistance mechanisms in ROS1-rearranged non-small cell lung cancer. Invest New Drugs. 2020;38(2):360-8.

29. Cocco E, Schram AM, Kulick A, et al. Resistance to TRK inhibition mediated by convergent MAPK pathway activation. Nat Med. 2019;25(9):1422-7.

30. Drilon A, Li G, Dogan S, et al. What hides behind the MASC: clinical response and acquired resistance to entrectinib after ETV6-NTRK3 identification in a mammary analogue secretory carcinoma (MASC). Ann Oncol. 2016;27(5):920-6.

31. Doebele RC, Dziadziuszko R, Drilon A, et al. Genomic landscape of entrectinib resistance from ctDNA analysis in STARTRK-2 [abstract no. LBA28]. Ann Oncol. 2019;30(Suppl 5):v865.

32. Tyler L, Le A, Nijmeh H, et al. MET mediates entrectinib resistance in ROS1 gene fusion positive NSCLC [abstract no. 3003]. In: AACR Annual Meeting; 2020.

33. Gonazlez-Sales M, Djebli N, Buchheit V, et al. Population pharmacokinetic (PK) analysis of entrectinib, a CNS-active, potent and selective inhibitor of ROS1/TRK/ALK kinase activity, in patients with solid tumors [abstract no. PIII-022]. Clin Pharmacol Ther. 2020;107(Suppl 1):S84-5.

34. Meneses-Lorente G, Bentley D, Guerini E, et al. Characterization of the pharmacokinetics of entrectinib and its active M5 metabolite in healthy volunteers and patients with solid tumors. Invest New Drugs. 2021. https://doi.org/10.1007/s10637-020-01047-5.

35. Doebele RC, Drilon A, Paz-Ares L, et al. Entrectinib in patients with advanced or metastatic NTRK fusion-positive solid tumours: integrated analysis of three phase 1-2 trials. Lancet Oncol. 2020;21(2):271-82.

36. Drilon A, Siena S, Dziadziuszko R, et al. Entrectinib in ROS1 fusion-positive non-small-cell lung cancer: integrated analysis of three phase 1-2 trials. Lancet Oncol. 2020;21(2):261-70.

37. Rolfo CD, De Braud FG, Doebele RC, et al. Efficacy and safety of entrectinib in patients (pts) with NTRK-fusion positive (NTRK-fp) solid tumors: an updated integrated analysis [abstract no. 3605 plus poster no. P335]. In: 56th ASCO Annual Meeting; 2020.

38. Dziadziuszko R, Siena S, Tan D, et al. Efficacy of entrectinib in patients with NTRK or ROS1 fusion-positive NSCLC with CNS metastases at baseline [abstract no. $1288 \mathrm{P}$ plus poster]. In: ESMO Virtual Congress; 2020.
39. Liu S, De Braud F, Drilon A, et al. Entrectinib in patients with ROS1 fusion-positive non-small cell lung cancer (NSCLC) or NTRK fusion-positive solid tumours: analysis of response by line of therapy. In: ESMO Virtual Congress; 2020.

40. Patel MR, Siena S, Demetri G, et al. Efficacy and safety of entrectinib in NTRK fusion-positive gastrointestinal cancers: updated integrated analysis of three clinical trials (STARTRK-2, STARTRK-1 and ALKA-372-001) [abstract no. O-3]. Ann Oncol. 2020;31(suppl 3):232-3.

41. Drilon A, Paz-Ares L, Doebele R, et al. Entrectinib in NTRK fusion-positive NSCLC: updated integrated analysis of patients in STARTRK-2, STARTRK-1 and ALKA-372-001 [abstract no. 543P plus poster]. In: ESMO Virtual Congress; 2020.

42. Conley AP, Demetri GD, Doebele RC, et al. Patient-reported outcomes (PROs) from patients (pts) with NTRK fusion-positive (NTRK-fp) solid tumours receiving entrectinib in the global phase II STARTRK-2 study [abstract no. 539P plus poster]. In: ESMO Virtual Congress; 2020.

43. Barlesi F, Wolf J, Ahn M-J, et al. Patient reported outcomes (PROs) analysis for patients with ROS1 fusion-positive (ROS1+) non-small cell lung cancer (NSCLC) receiving entrectinib in the global phase II STARTRK-2 study [abstract no. 385P]. Ann Oncol. 2020;31(Suppl 6):S1391-2

44. Tan DSW, Ahn M-J,, Chiu C-H, et al. Efficacy and safety of entrectinib in an Asian population with NTRK fusion-positive (fp) solid tumours or ROS1-fp NSCLC [abstract no. 299MO]. Ann Oncol. 2020;31(Suppl 6):S1358-9.

45. Robinson G, Desai A, Basu E, et al. Entrectinib in recurrent or refractory solid tumors including primary CNS tumors: updated data in children and adolescents [abstract HGG-01 plus oral presentation]. Neuro Oncol. 2020;22(Suppl 3):iii344.

46. European Medicines Agency. Vitrakvi 25 and $100 \mathrm{mg}$ hard capsules: EU summary of product characteristics. 2020. https://www. ema.europa.eu. Accessed 25 Mar 2021.

47. National Comprehensive Cancer Network. NCCN guidelines. 2020. https://www.nccn.org. Accessed 25 Mar 2021.

48. Hong DS, DuBois SG, Kummar S, et al. Larotrectinib in patients with TRK fusion-positive solid tumours: a pooled analysis of three phase 1/2 clinical trials. Lancet Oncol. 2020;21(4):531-40.

49. Drilon A. TRK inhibitors in TRK fusion-positive cancers. Ann Oncol. 2019;30(Suppl 8):viii23-30.

50. Huang L, Jiang S, Shi Y. Tyrosine kinase inhibitors for solid tumors in the past 20 years (2001-2020). J Hematol Oncol. 2020;13:143.

51. Ardini E, Siena S. Entrectinib approval by EMA reinforces options for ROS1 and tumour agnostic NTRK targeted cancer therapies. ESMO Open. 2020;5:e000867.

52. Chu P, Antoniou M, Bhutani MK, et al. Matching-adjusted indirect comparison: entrectinib versus crizotinib in ROS1 fusion-positive non-small cell lung cancer. J Comp Eff Res. 2020;9(12):861-76.

53. Doebele R, Perez L, Trinh $\mathrm{H}$, et al. Comparative efficacy analysis between entrectinib trial and crizotinib real-world ROS1 fusionpositive $(R O S 1+)$ NSCLC patients [abstract no. P1.01-83]. J Thorac Oncol. 2019;14(10 Suppl):S392.

54. Sehgal K, Piper-Vallillo AJ, Viray H, et al. Cases of ROS1-rearranged lung cancer: when to use crizotinib, entrectinib, lorlatinib, and beyond? Precis Cancer Med. 2020;3:17.

55. Dziadziuszko R, André F, Yip W-K, et al. Clinical validity of FoundationOne liquid CDx (F1L CDx) assay as an aid in selecting patients for treatment with entrectinib [abstract no. 1193P plus poster]. In: ESMO Virtual Congress; 2020.

56. Foundation Medicine, Inc. https://www.foundationmedicine.com/ test/foundationone-liquid-cdx. Accessed 25 Mar 2021. 